\title{
Optimal Control of a Delay-Varying Computer Virus Propagation Model
}

\author{
Jianguo Ren, ${ }^{1}$ Yonghong $\mathrm{Xu},{ }^{2}$ and Chunming $\mathrm{Zhang}^{3}$ \\ ${ }^{1}$ College of Computer, Jiangsu Normal University, Jiangsu 221116, China \\ ${ }^{2}$ College of Bioengineering, Chongqing University, Chongqing 400044, China \\ ${ }^{3}$ School of Information Engineering, Guangdong Medical College, Dongguan 523808, China
}

Correspondence should be addressed to Jianguo Ren; rjgrjgrjgrjg@126.com

Received 1 April 2013; Revised 20 July 2013; Accepted 21 July 2013

Academic Editor: Xiaofan Yang

Copyright (C) 2013 Jianguo Ren et al. This is an open access article distributed under the Creative Commons Attribution License, which permits unrestricted use, distribution, and reproduction in any medium, provided the original work is properly cited.

\begin{abstract}
By incorporating the objective of keeping a low number of infected nodes and a high number of recovered nodes at a lower cost into a known computer virus model (the delay-varying SIRC model) extended by introducing quarantine, a novel model is described by means of the optimal control strategy and theoretically analyzed. Through the comparison of simulation results, it is shown that the propagation of computer virus with varying latency period can be suppressed effectively by the optimal control strategy.
\end{abstract}

\section{Introduction}

With the advance of computer software and hardware technology and communication technology, the number and sort of computer viruses have increased dramatically, which causes huge losses to the human society. Therefore, establishing reasonable computer virus propagation models by considering the characteristics of computer virus, by model analysis, and by understanding the spread law of the virus over the network is a currently hot topic of research.

Learning from the epidemic models, the classical SIR (susceptible-infected-recovered) computer virus propagation model $[1,2]$, as well as its extensions [3-15], is extended to explore the behavior of computer virus propagation in network. For example, by considering the computer virus fixed latent period, Mishra et al. [6,7] proposed delayed SIRS, SEIR computer virus models with a fixed period of temporary immunity, which accounts for the temporary recovery from the infection of virus. Very recently, Ren and $\mathrm{Xu}$ [16] introduced an interesting virus propagation model, known as the SIRC model, by considering the fact that when the virus enters into the susceptible computers, their latency periods vary and investigated the dynamics of the model. Once the rule of the virus spread is revealed, it comes to be a major issue how to control the virus spread effectively [17-19].
In this paper, by incorporating the objective of keeping a low number of infected nodes and a high number of recovered nodes at the lower cost into a delay-varying computer virus propagation model (SIRC model) extended by introducing the new compartment quarantine, a novel model is described by means of the optimal control strategies associated with measures of quarantine and installing antivirus programs and firewalls and theoretically analyzed. It is comparatively showed that optimal control strategy is much more effective for controlling virus with varying latency period in network.

The remaining materials of this paper are organized this way: Section 2 introduces the mathematical model to be discussed; Section 3 studies the controlled system theoretically. In Section 4, we solve the controlled system numerically using the Runge-Kutta procedure and make numerical comparisons with control and without control. We end the paper with a brief conclusion in Section 5 .

\section{Mathematical Model}

Consider a delay-varying computer virus model recently proposed in [16]

$$
\frac{d S}{d t}=b-\beta \int_{-\infty}^{t} S(\tau) K(t-\tau) d \tau I(t)-\mu S(t),
$$




$$
\begin{gathered}
\frac{d I}{d t}=\beta \int_{-\infty}^{t} S(\tau) K(t-\tau) d \tau I(t)-(\mu+\gamma) I(t), \\
\frac{d R}{d t}=\gamma I(t)-\mu R(t) .
\end{gathered}
$$

Here, it is assumed that all the computers connected to the network in concern are classified into three categories: susceptible, infected, and recovered computers. Let $S(t), I(t)$, and $R(t)$ denote their corresponding numbers at time $t$. This model involves some positive parameters: $b$ denotes the rate at which external computers are connected to the network, $\gamma$ denotes the recovery rate of infected computers due to the antivirus ability of the network, $\mu$ denotes the rate at which one computer is removed from the network, and $\beta$ denotes the rate at which, when having connection to one infected computer, one susceptible computer can become infected. By appropriate assumptions and extensions in [16], model (1) can be written as

$$
\begin{gathered}
\frac{d S}{d t}=b-\beta C(t) I(t)-\mu S(t), \\
\frac{d I}{d t}=\beta C(t) I(t)-(\mu+\gamma) I(t), \\
\frac{d R}{d t}=\gamma I(t)-\mu R(t), \\
\frac{d C}{d t}=\frac{1}{\sigma} S(t)-\frac{1}{\sigma} C(t),
\end{gathered}
$$

where $C(t)$ denotes the effect of past infection information in the susceptible computers at time $t, \sigma$ is a positive delay parameter with the initial conditions $S(0) \geq 0, I(0) \geq 0$, $C(0) \geq 0$, and the positively invariant set

$$
\Omega=\left\{(S, I, C) \in R_{+}^{3}, S+I \leq \frac{b}{\mu}, C \leq \frac{b}{\mu}\right\} .
$$

Recently, more research attention has been paid to the combination of virus propagation models and antivirus countermeasures to investigate the prevalence of virus. As an elementary measure, quarantine $[20,21]$ is used to restrain the spread of computer virus. Extending the previous SIRC, a new compartment quarantine has been introduced under which the susceptible, infected computers exhibited suspicious behavior and, consequently, have been quarantined. The model with quarantine can be written as

$$
\begin{gathered}
\frac{d S}{d t}=b-\beta C(t) I(t)-\alpha S(t)-\mu S(t), \\
\frac{d I}{d t}=\beta C(t) I(t)-(\mu+\gamma) I(t)-\varepsilon I(t), \\
\frac{d R}{d t}=\gamma I(t)-\mu R(t), \\
\frac{d Q}{d t}=\varepsilon I(t)+\alpha S(t)-\mu Q(t), \\
\frac{d C}{d t}=\frac{1}{\sigma} S(t)-\frac{1}{\sigma} C(t),
\end{gathered}
$$

where parameter $\alpha$ denotes the rate at which the susceptible computers are quarantined, $\varepsilon$ denotes the rate at which the infected computers are quarantined.

For our purpose, first, we introduce two Lebesgue square integrable control functions.

(1) $u_{1}(t)$ denotes the cost for installing effective antivirus programs and firewalls on the susceptible computers and infected computers at time $t$.

(2) $u_{2}(t)$ denotes the cost for quarantining the susceptible and infected computers at time $t$.

Both of the control functions are normalized to fall between 0 and 1 , and the admissible set of control functions is given by

$$
\begin{gathered}
U_{\mathrm{ad}}=\left\{u_{1}(t), u_{2}(t) \in L^{2}: 0 \leq u_{1}(t) \leq 1,\right. \\
\left.0 \leq u_{2}(t) \leq 1, t \in\left[0, t_{f}\right]\right\} .
\end{gathered}
$$

To obtain the controlled model, the following assumptions are made.

(1) At time $t$, there are $p u_{1}(t) I(t)$ infected computers that would become recovered, whereas there are (1 p) $u_{1}(t) I(t)$ that would be quarantined, where $p \in$ $[0,1]$.

(2) At time $t$, by installing antivirus programs and firewalls, there are $q u_{2}(t) S(t)$ susceptible computers that would directly become recovered, whereas there are $(1-q) u_{2}(t) S(t)$ that would be quarantined, where $q \epsilon$ $[0,1]$.

Taking into account the assumptions made above, the model (7) can become the following computer virus propagation model:

$$
\begin{gathered}
\frac{d S}{d t}=b-\beta C(t) I(t)-\alpha S(t)-\mu S(t)-u_{2} S(t), \\
\frac{d I}{d t}=\beta C(t) I(t)-(\mu+\gamma) I(t)-\varepsilon I-u_{1} I(t), \\
\frac{d R}{d t}=\gamma I(t)-\mu R(t)+p u_{1} I(t)+q u_{2} S(t), \\
\frac{d Q}{d t}=\varepsilon I(t)+\alpha S(t)+(1-p) u_{1} I(t) \\
+(1-q) u_{2} S(t)-\mu Q(t) \\
\frac{d C}{d t}=\frac{1}{\sigma} S(t)-\frac{1}{\sigma} C(t)
\end{gathered}
$$

with the given initial conditions and the positively invariant set.

\section{Optimal Control Problem}

During the time period $\left[0, t_{f}\right]$, under the above assumptions and extensions, our objective is given by the following.

(1) Minimize the number of infected computers $(I)$ and maximize the number of recovered computers $(R)$. 
(2) Minimize the total cost to quarantine the susceptible and infected computers.

(3) Minimize the total cost for installing the antivirus programs and firewalls in the susceptible and infected computers.

Thus, our optimal control problem is to minimize the objective functional:

$$
J(u)=\int_{0}^{t_{f}}\left[I(t)+\eta C(t)+\frac{1}{2} \tau_{1} u^{2}(t)+\frac{1}{2} \tau_{2} u_{2}^{2}(t)\right] d t,
$$

where parameter $\eta$ denotes the weight constants of effect of latent virus, $\tau_{1}, \tau_{2}$ are trade-off factors.

To find an optimal solution to (4), consider the Lagrangian

$$
L(I, C, u)=I(t)+\eta C(t)+\frac{1}{2} \tau_{1} u^{2}(t)+\frac{1}{2} \tau_{2} u_{2}^{2}(t) .
$$

Define the Hamiltonian $H$ for the control problem as

$$
\begin{aligned}
H(I, C, & \left.u, \lambda_{1}, \lambda_{2}, \lambda_{3}, \lambda_{4}, \lambda_{5}\right) \\
= & L(I, C, u)+\lambda_{1}(t) \frac{d S}{d t}+\lambda_{2}(t) \frac{d I}{d t} \\
& +\lambda_{3}(t) \frac{d R}{d t}+\lambda_{4}(t) \frac{d Q}{d t}+\lambda_{5}(t) \frac{d C}{d t} \\
= & I(t)+\eta C(t)+\frac{1}{2} \tau_{1} u^{2}(t)+\frac{1}{2} \tau_{2} u_{2}^{2}(t) \\
+ & \lambda_{1}(t)\{b-\beta C(t) I(t) \\
& \left.-\alpha S(t)-\mu S(t)-u_{2} S(t)\right\} \\
+ & \lambda_{2}(t)\{\beta C(t) I(t)-(\mu+\gamma+\varepsilon) \\
& \left.\times I(t)-u_{1} I(t)\right\} \\
+ & \lambda_{3}(t)\{\gamma I(t)-\mu R(t) \\
& \left.+p u_{1} I(t)+q u_{2} S(t)\right\} \\
+ & \lambda_{4}(t)\{\varepsilon I(t)+\alpha S(t)-\mu Q(t) \\
& +(1-p) u_{1} \times I(t) \\
& \left.+(1-q) u_{2} S(t)\right\} \\
+ & \lambda_{5}(t)\left\{\frac{1}{\sigma}(S-C)\right\},
\end{aligned}
$$

with the transversality conditions (or boundary conditions) $\lambda_{i}\left(t_{f}\right)=0, i=1,2,3,4,5$.

Theorem 1. There exist control functions $u_{1}^{*}, u_{2}^{*}$ so that $J\left(u_{1}^{*}, u_{2}^{*}\right)=\min _{u_{1}, u_{2} \in U_{\text {ad }}} J\left(u_{1}, u_{2}\right)$ subject to the controlled system (6) with initial condition.

Proof. We use the results in $[22,23]$. It is clear that the set of control and corresponding state variables are nonnegative values and the set $U_{\text {ad }}$ is convex and closed.

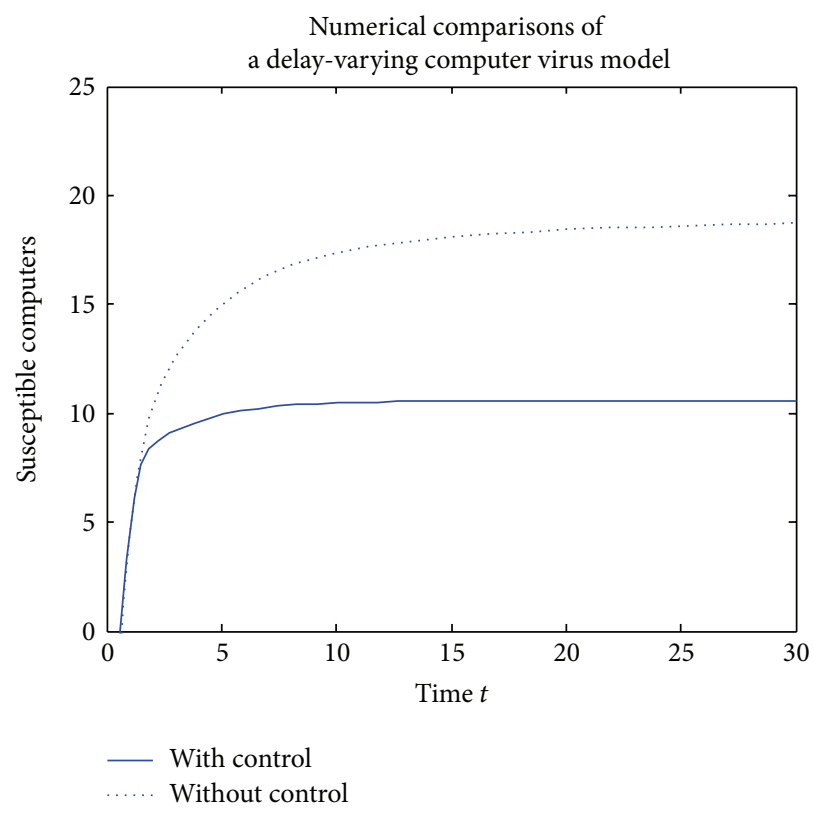

FIGURE 1: Susceptible computers (with and without control) versus time $t$.

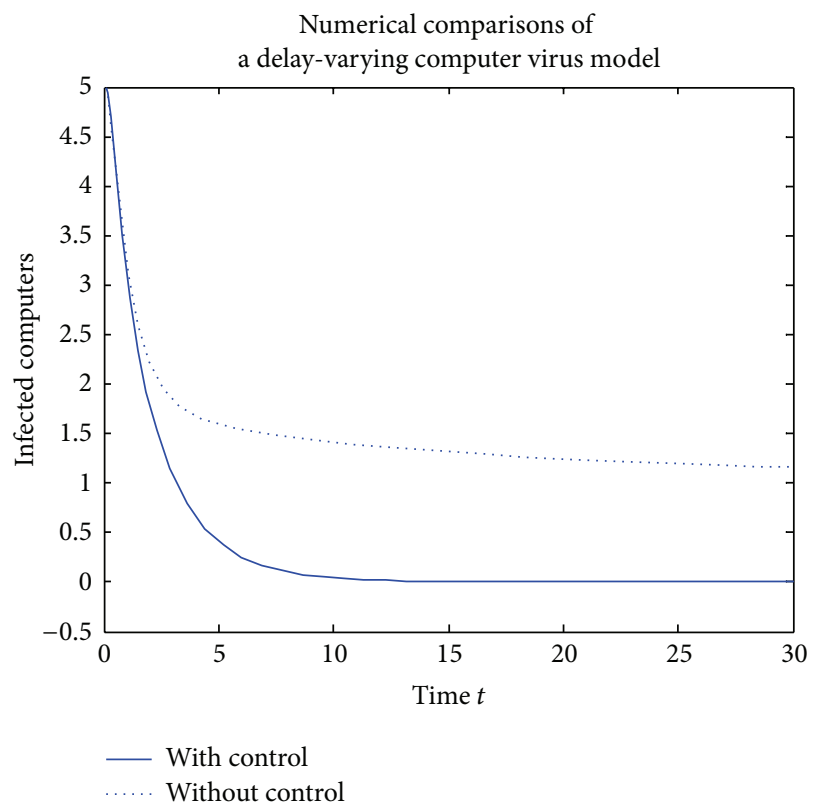

FIGURE 2: Infected computers (with and without control) versus time $t$.

$L(I, C, u)=I(t)+\eta C(t)+(1 / 2) \tau_{1} u^{2}(t)+(1 / 2) \tau_{2} u_{2}{ }^{2}(t)$ is convex on $U_{\mathrm{ad}}$. Meanwhile, the optimal system (4) is bounded by a linear function in the state variables. Also, there exist a constant $\varsigma>1$ and two positive numbers $\sigma_{1}$ and $\sigma_{2}$ so that $L(I, C, u) \geq \sigma_{1}+\sigma_{1}\left(\left|u_{1}\right|+\left|u_{2}\right|\right)^{\varsigma / 2}$.

In the following, we use Pontryagin's maximum principle [24] to obtain a necessary condition for the optimal control solution to the system (4). 
TABle 1

\begin{tabular}{lcccccccccccc}
\hline Parameters & $b$ & $\beta$ & $\gamma$ & $\alpha$ & $\varepsilon$ & $\mu$ & $\sigma$ & $p$ & $q$ & $\eta$ & $\tau_{1}$ & $\tau_{2}$ \\
\hline Values & 20 & 0.05 & 0.25 & 0.5 & 0.2 & 0.5 & 0.5 & 0.3 & 0.4 & 0.8 & 30 & 50 \\
\hline
\end{tabular}

TABle 2

\begin{tabular}{lccccc}
\hline Initial state variable & $S(0)$ & $I(0)$ & $R(0)$ & $Q(0)$ & $C(0)$ \\
\hline Values & 0 & 5 & 4.5 & 13 & 8 \\
\hline
\end{tabular}

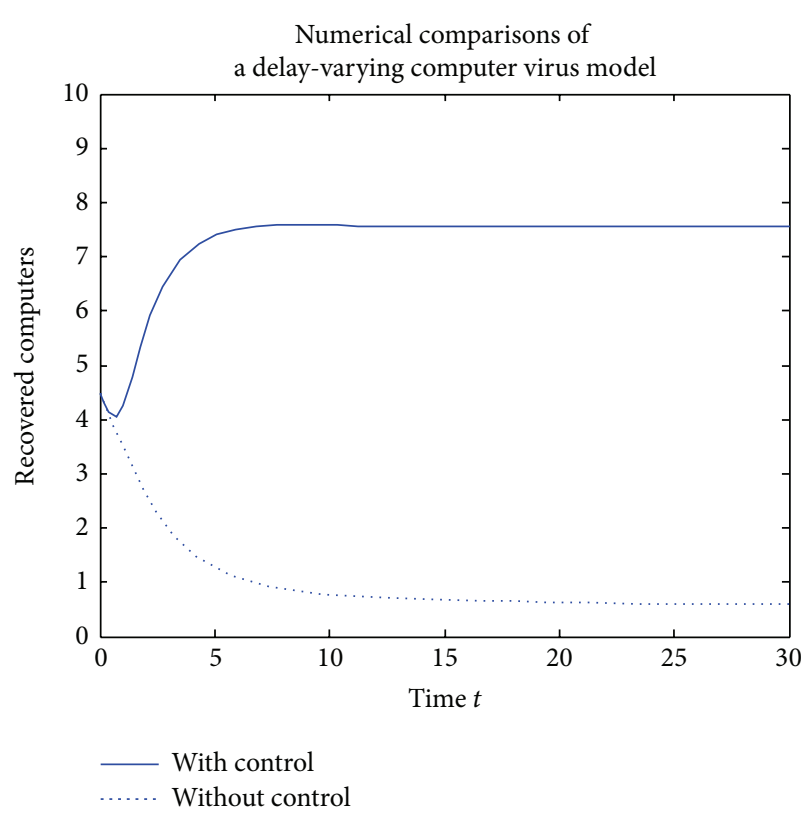

FIGURE 3: Recovered computers (with and without control) versus time $t$.

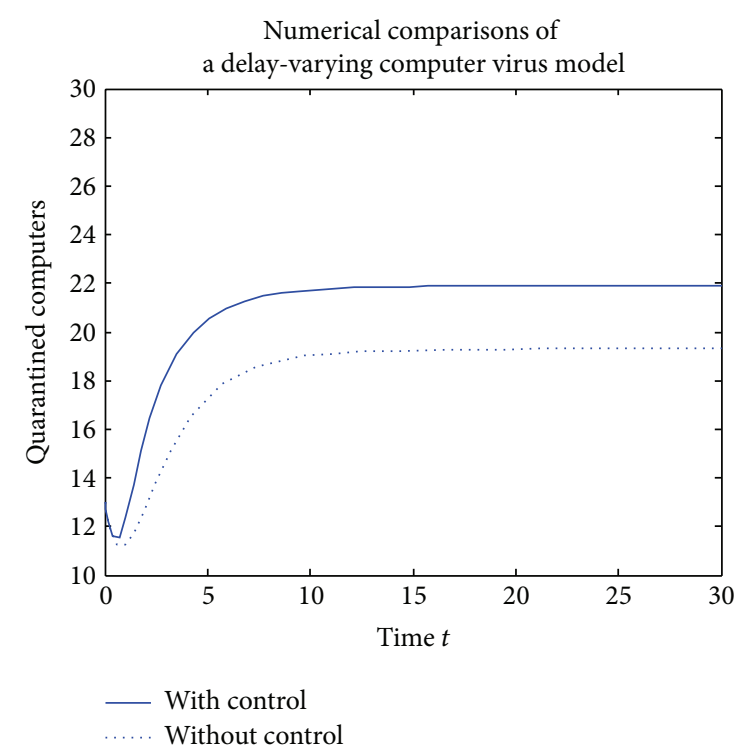

FIGURE 4: Quarantined computers (with and without control) versus time $t$.

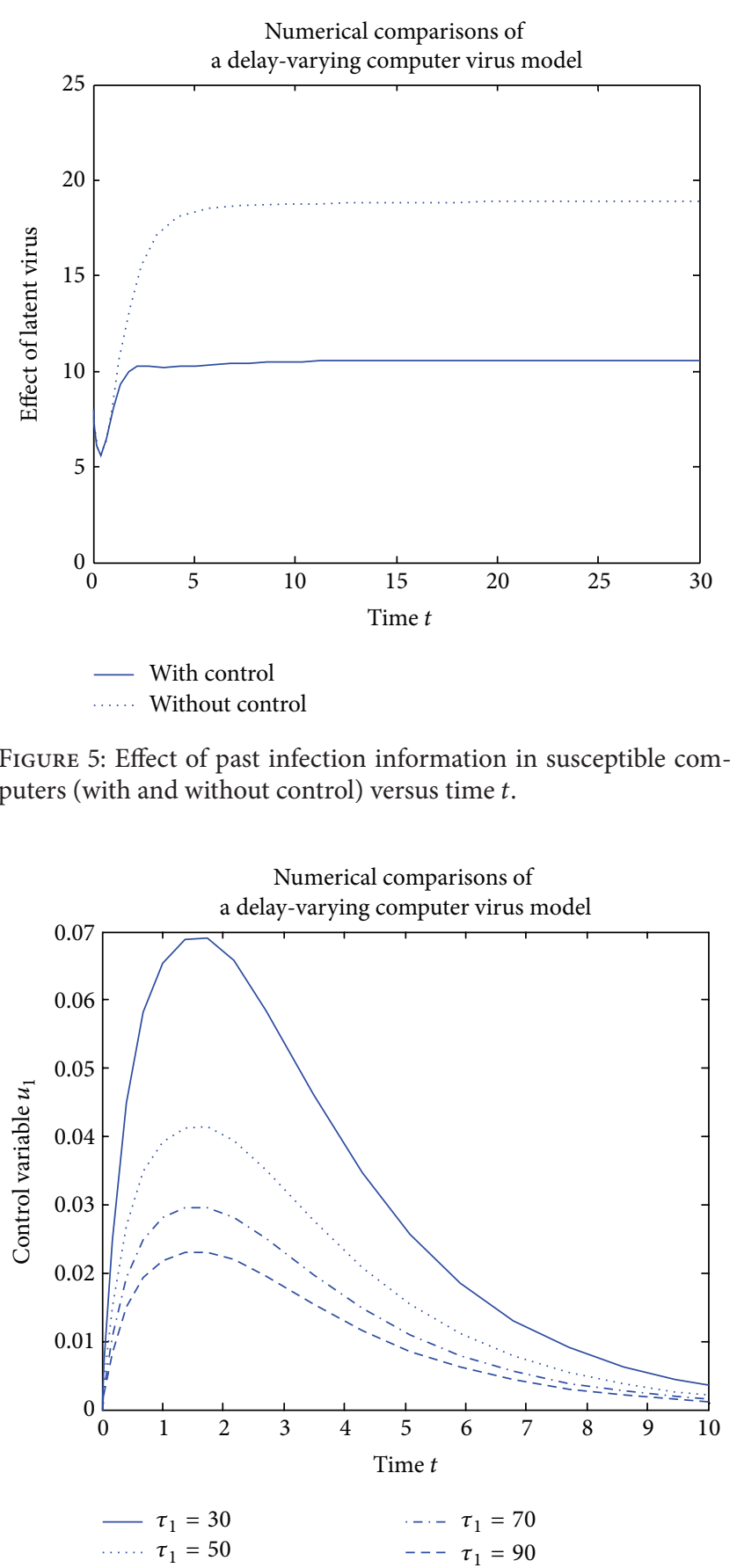

FIGURE 6: The control variable $u_{1}(t)$ versus time $t$ for different weight factors. 


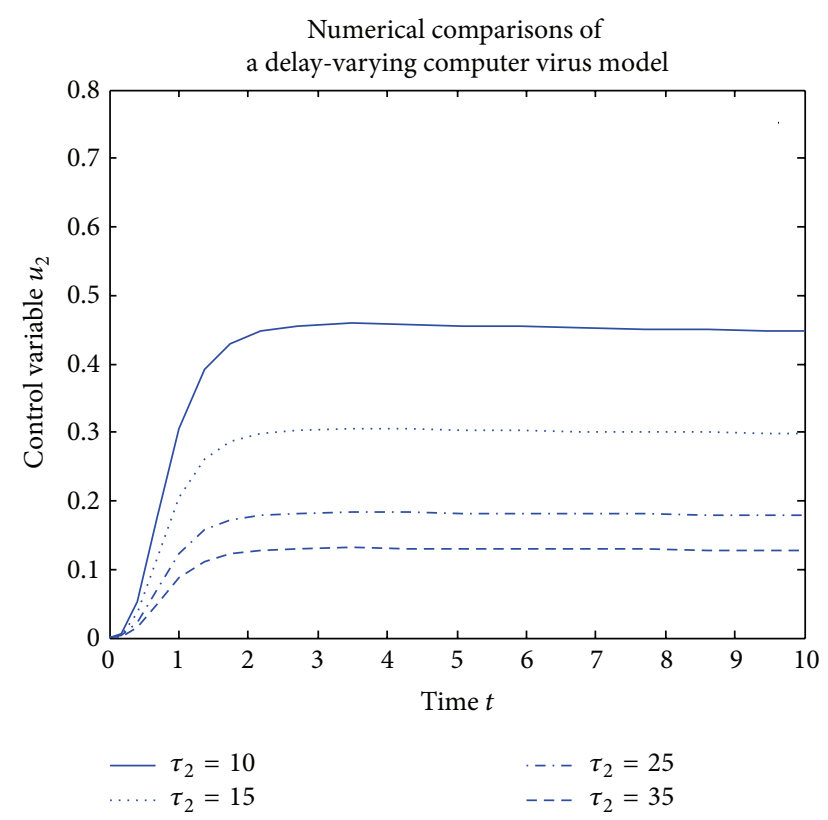

Figure 7: The control variable $u_{2}(t)$ versus time $t$ for different weight factors.

Theorem 2. Consider the optimal control problem (7) subject to (6). Let $S^{*}(t), I^{*}(t), R^{*}(t), Q^{*}(t)$, and $C^{*}(t)$ form the optimal state solution with associated optimal control variables $u_{1}^{*}(t), u_{2}^{*}(t)$. Then, there exist adjoint variables $\lambda_{1}(t)$, $\lambda_{2}(t), \lambda_{3}(t), \lambda_{4}(t)$, and $\lambda_{5}(t)$ that satisfy

$$
\begin{gathered}
\frac{d \lambda_{1}(t)}{d t}=\lambda_{1}\left(\mu+\alpha+u_{2}\right)-\lambda_{3} q u_{2}-\lambda_{4}(1-q) u_{2}-\lambda_{5} \frac{1}{\sigma} \\
\frac{d \lambda_{2}(t)}{d t}=-\lambda_{1} \beta C-\lambda_{2}\left(\beta C-\gamma-\mu-\varepsilon-u_{1}\right) \\
-\lambda_{3}\left(\gamma+p u_{1}\right)-\lambda_{4}\left\{\varepsilon+(1-p) u_{1}\right\}-1 \\
\frac{d \lambda_{3}(t)}{d t}=\lambda_{3} \mu \\
\frac{d \lambda_{4}(t)}{d t}=\lambda_{4} \mu, \\
\frac{d \lambda_{5}(t)}{d t}=\lambda_{1} \beta I-\lambda_{2} \beta I+\lambda_{5} \frac{1}{\sigma}-\eta
\end{gathered}
$$

with transversality conditions $\lambda_{i}\left(t_{f}\right)=0, i=1,2,3,4,5$.

Proof. By the adjoint equations and optimality conditions, we have

$$
\begin{aligned}
\frac{d \lambda_{1}(t)}{d t}= & -\frac{\partial H}{\partial S} \\
= & \lambda_{1}\left(\mu+\alpha+u_{2}\right)-\lambda_{3} q u_{2} \\
& -\lambda_{4}(1-q) u_{2}-\lambda_{5} \frac{1}{\sigma}
\end{aligned}
$$

$$
\begin{gathered}
\frac{d \lambda_{2}(t)}{d t}=-\frac{\partial H}{\partial I} \\
=-\lambda_{1} \beta C-\lambda_{2}\left(\beta C-\gamma-\mu-\varepsilon-u_{1}\right) \\
-\lambda_{3}\left(\gamma+p u_{1}\right)-\lambda_{4}\left\{\varepsilon+(1-p) u_{1}\right\}-1, \\
\frac{d \lambda_{3}(t)}{d t}=-\frac{\partial H}{\partial R}=\lambda_{3} \mu \\
\frac{d \lambda_{4}(t)}{d t}=-\frac{\partial H}{\partial Q}=\lambda_{4} \mu, \\
\frac{d \lambda_{5}(t)}{d t}=-\frac{\partial H}{\partial C}=\lambda_{1} \beta I-\lambda_{2} \beta I+\lambda_{5} \frac{1}{\sigma}-\eta \\
\tau u_{1}^{*}(t)-\lambda_{2}(t) I^{*}+\lambda_{3}(t) p I^{*}+\lambda_{4}(t)(1-p) I^{*}=0 \\
\tau u_{2}^{*}(t)-\lambda_{1}(t) S^{*}+\lambda_{3}(t) q S^{*}+\lambda_{4}(t)(1-q) S^{*}=0
\end{gathered}
$$

which can be obtained from $\partial H / \partial u_{1}=0, \partial H / \partial u_{2}=0$, respectively.

Noting a fact that $0 \leq u_{1}(t) \leq 1,0 \leq u_{2}(t) \leq 1$, we obtain that

$u_{1}^{*}(t)=\max \left\{\min \left\{\frac{\left(\lambda_{2}(t)-\lambda_{3}(t) p-\lambda_{4}(t)(1-p)\right) I^{*}}{\tau_{1}}\right.\right.$,

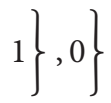

$u_{2}^{*}(t)=\max \left\{\min \left\{\frac{\left(\lambda_{1}(t)-\lambda_{3}(t) q-\lambda_{4}(t)(1-q)\right) S^{*}}{\tau_{2}}\right.\right.$, $1\}, 0\}$.

From the previous analysis, to get the optimal point, we have to solve the system

$$
\begin{gathered}
\frac{d S^{*}}{d t}=b-\beta C^{*} I^{*}-\mu S^{*}-\alpha S^{*}-u_{2} S^{*}, \\
\frac{d I^{*}}{d t}=\beta C^{*} I^{*}-(\mu+\gamma) I^{*}-\varepsilon I^{*}-u_{1}^{*} I^{*}, \\
\frac{d R^{*}}{d t}=\gamma I^{*}-\mu R^{*}+p u_{1}^{*} I^{*}+q u_{2}^{*} S^{*}, \\
\frac{d Q^{*}}{d t}=\alpha S^{*}+\varepsilon I^{*}+(1-p) u_{1}^{*} I^{*}+(1-q) u_{2}^{*} S^{*}-\mu Q^{*}, \\
\frac{d C^{*}}{d t}=\frac{1}{\sigma}\left(S^{*}-C^{*}\right),
\end{gathered}
$$


with the Hamiltonian

$$
\begin{aligned}
H^{*}\left(I^{*}, C^{*}, u^{*}, \lambda_{1}, \lambda_{2}, \lambda_{3}, \lambda_{4}, \lambda_{5}\right) \\
=I^{*}+\eta C^{*}+\frac{\tau}{2} u_{1}^{*}(t)^{2} \\
+\frac{\tau}{2} u_{2}^{*}(t)^{2}+\lambda_{1}(t) \\
\times\left\{b-\beta C^{*} I^{*}-\alpha S^{*}\right. \\
\left.\quad-\mu S^{*}-u_{2}^{*}(t) S^{*}\right\}+\lambda_{2}(t) \\
\times\left\{\beta C^{*} I^{*}-(\mu+\gamma-\varepsilon)\right. \\
\left.\quad \times I^{*}-u_{1}^{*}(t) I^{*}\right\} \\
+\lambda_{3}(t)\left\{\gamma I^{*}-\mu R^{*}\right. \\
\left.\quad+p u_{1}^{*}(t) I^{*}+q u_{2}^{*}(t) S^{*}\right\} \\
+\lambda_{4}(t)\left\{\gamma I^{*}+\alpha S^{*}-\mu Q^{*}\right. \\
\quad+(1-p) u_{1}^{*}(t) I^{*}+(1-q) u_{2}^{*} \\
\left.\quad \times(t) S^{*}\right\}+\lambda_{5}(t)\left\{\frac{1}{\sigma}\left(S^{*}-C^{*}\right)\right\}
\end{aligned}
$$

\section{Numerical Simulations}

In this section, to find out the optimal control solution, we numerically solve system (13) with (14) by a Runge-Kutta procedure and make numerical comparisons. Figures 1-5 plot the numbers of susceptible, infected, recovered, and quarantined computers as well as the effect of past infection information in the susceptible computers with and without control, with the parameters shown in Table 1 and the initial conditions shown in Table 2.

The number of computers under control is marked by solid line, whereas the number of remaining computers is marked by dashed line. One can see that, under control, the number of susceptible computers is sharply low, which is the same as the effect of past infection information in Figure 5. In Figure 2, we can see that the number of infected computers under control is lower than that without control. In Figure 3, the number of recovered computers is very small without control and more recovered computers increase more rapidly. Although the number of quarantined computers with control would slightly grow in Figure 4, we mainly consider tradeoff between the cost and effect of the quarantine. Figures 6 and 7 plot the control variables $u_{1}, u_{2}$ versus time $t$ with associated weight factor $\tau_{1} \in\{30,50,70,90\}$ and $\tau_{2} \in\{10,15,25,35\}$, respectively.

\section{Conclusions}

By incorporating the objective of keeping a low number of infected nodes and a high number of recovered nodes at the lower cost into a known computer virus model (the delayvarying SIRC model) extended by introducing quarantine, a novel model is described by means of the optimal control strategy and theoretically analyzed. A comparison between optimal control and without control is presented, which demonstrates the effectiveness of our method. The results obtained in the present paper can help understand and control the spread of computer virus over a computer network.

\section{Acknowledgments}

The work is supported by the National Natural Science Foundation of China under Grant no. 61304117, no. 61272297 and no. 61100167, the Natural Science Foundation of Jiangsu Province, China under Grant no. BK2011204, the doctorate teacher support project of JiangSu Normal University under Grant no. 12XLR021, and the Natural Science Foundation of the Jiangsu Higher Education Institutions of China under Grant no. 13KJB520008, the Natural Science Foundation of the Jiangsu Higher Education Institutions of China under Grant no. 11KJB520019.

\section{References}

[1] J. C. Wierman and D. J. Marchette, "Modeling computer virus prevalence with a susceptible-infected-susceptible model with reintroduction," Computational Statistics \& Data Analysis, vol. 45, no. 1, pp. 3-23, 2004.

[2] J. R. C. Piqueira and V. O. Araujo, "A modified epidemiological model for computer viruses," Applied Mathematics and Computation, vol. 213, no. 2, pp. 355-360, 2009.

[3] C. Gan, X. Yang, W. Liu, Q. Zhu, and X. Zhang, "Propagation of computer virus under human intervention: a dynamical model," Discrete Dynamics in Nature and Society, vol. 2012, Article ID 106950, 8 pages, 2012.

[4] Q. Zhu, X. Yang, and J. Ren, "Modeling and analysis of the spread of computer virus," Communications in Nonlinear Science and Numerical Simulation, vol. 17, no. 12, pp. 5117-5124, 2012.

[5] L.-X. Yang, X. Yang, J. Liu, Q. Zhu, and C. Gan, "Epidemics of computer viruses: s complex-network approach," Applied Mathematics and Computation, vol. 219, no. 16, pp. 8705-8717, 2013.

[6] B. K. Mishra and D. K. Saini, "SEIRS epidemic model with delay for transmission of malicious objects in computer network," Applied Mathematics and Computation, vol. 188, no. 2, pp. 14761482, 2007.

[7] B. K. Mishra and N. Jha, "Fixed period of temporary immunity after run of anti-malicious software on computer nodes," Applied Mathematics and Computation, vol. 190, no. 2, pp. 12071212, 2007.

[8] L.-X. Yang and X. Yang, "The spread of computer viruses under the influence of removable storage devices," Applied Mathematics and Computation, vol. 219, no. 8, pp. 3914-3922, 2012.

[9] L.-X. Yang, X. Yang, L. Wen, and J. Liu, "A novel computer virus propagation model and its dynamics," International Journal of Computer Mathematics, vol. 89, no. 17, pp. 2307-2314, 2012.

[10] J. Ren, X. Yang, Q. Zhu, L.-X. Yang, and C. Zhang, "A novel computer virus model and its dynamics," Nonlinear Analysis: Real World Applications, vol. 13, no. 1, pp. 376-384, 2012. 
[11] J. Ren, X. Yang, L.-X. Yang, Y. Xu, and F. Yang, "A delayed computer virus propagation model and its dynamics," Chaos, Solitons \& Fractals, vol. 45, no. 1, pp. 74-79, 2012.

[12] L.-X. Yang, X. Yang, Q. Zhu, and L. Wen, "A computer virus model with graded cure rates," Nonlinear Analysis: Real World Applications, vol. 14, no. 1, pp. 414-422, 2013.

[13] X. Yang, B. K. Mishra, and Y. Liu, "Theory, model and methods," Discrete Dynamics in Nature and Society, vol. 2012, Article ID 473508, 2 pages, 2012.

[14] X. Yang and L. -X. Yang, "Towards the epidemiological modeling of computer viruses," Discrete Dynamics in Nature and Society, vol. 2012, Article ID 259671, 11 pages, 2012.

[15] L.-X. Yang and X. Yang, "Propagation behavior of virus codes in the situation that infected computers are connected to the internet with positive probability," Discrete Dynamics in Nature and Society, vol. 2012, Article ID 693695, 13 pages, 2012.

[16] J. Ren and Y. Xu, "Dynamics of a delay-varying computer virus propagation model," Discrete Dynamics in Nature and Society, vol. 2012, Article ID 372192, 12 pages, 2012.

[17] C. Zhang, X. Yang, and Q. Zhu, "An optimal control model for computer viruses," Journal of Information and Computational Science, vol. 8, no. 13, pp. 2587-2596, 2011.

[18] Q. Zhu and X. Yang, "Optimal control of computer virus under a delayed model," Applied Mathematics and Computation, vol. 218, no. 23, pp. 11613-11619.

[19] J. Ren and X. Yang, "Dynamics and optimal shelter for computer virus propagation in network," Journal of Information and Computational Science, vol. 8, no. 9, pp. 1735-1745, 2011.

[20] D. Moore, C. Shannon, G. M. Voelker, and S. Savage, "Internet quarantine: requirements for containing self-propagating code," in Proceedings of the 22nd IEEE Annual Joint Conference on the Computer and Communications Societies (INFOCOM '03), pp. 1901-1910, IEEE, April 2003.

[21] T. M. Chen and N. Jamil, "Effectiveness of quarantine in worm epidemics," in Proceedings of the IEEE International Conference on Communications (ICC '05), pp. 2142-2147, IEEE, July 2005.

[22] W. H. Fleming and R. W. Rishel, Deterministic and Stochastic, Springer, Berlin, Germany, 1975.

[23] D. L. Lukes, Differential Equations: Classical to Controlled, vol. 162 of Mathematics in Science and Engineering, Academic Press, New York, NY, USA, 1982.

[24] M. L. Kamien and N. L. Schwartz, Dynamics Optimization: The Clculus of Variations and Optimal Control in Economics and Management, Elsevier Science, Amsterdam, The Netherlands, 2000. 


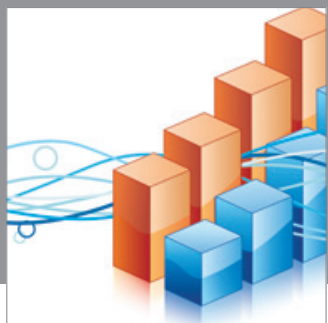

Advances in

Operations Research

mansans

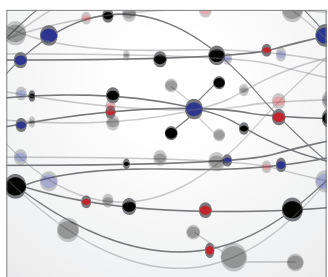

The Scientific World Journal
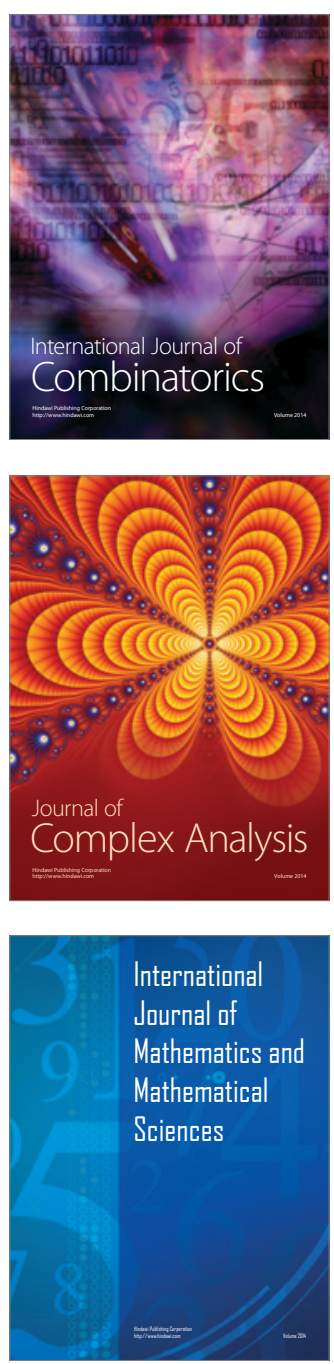
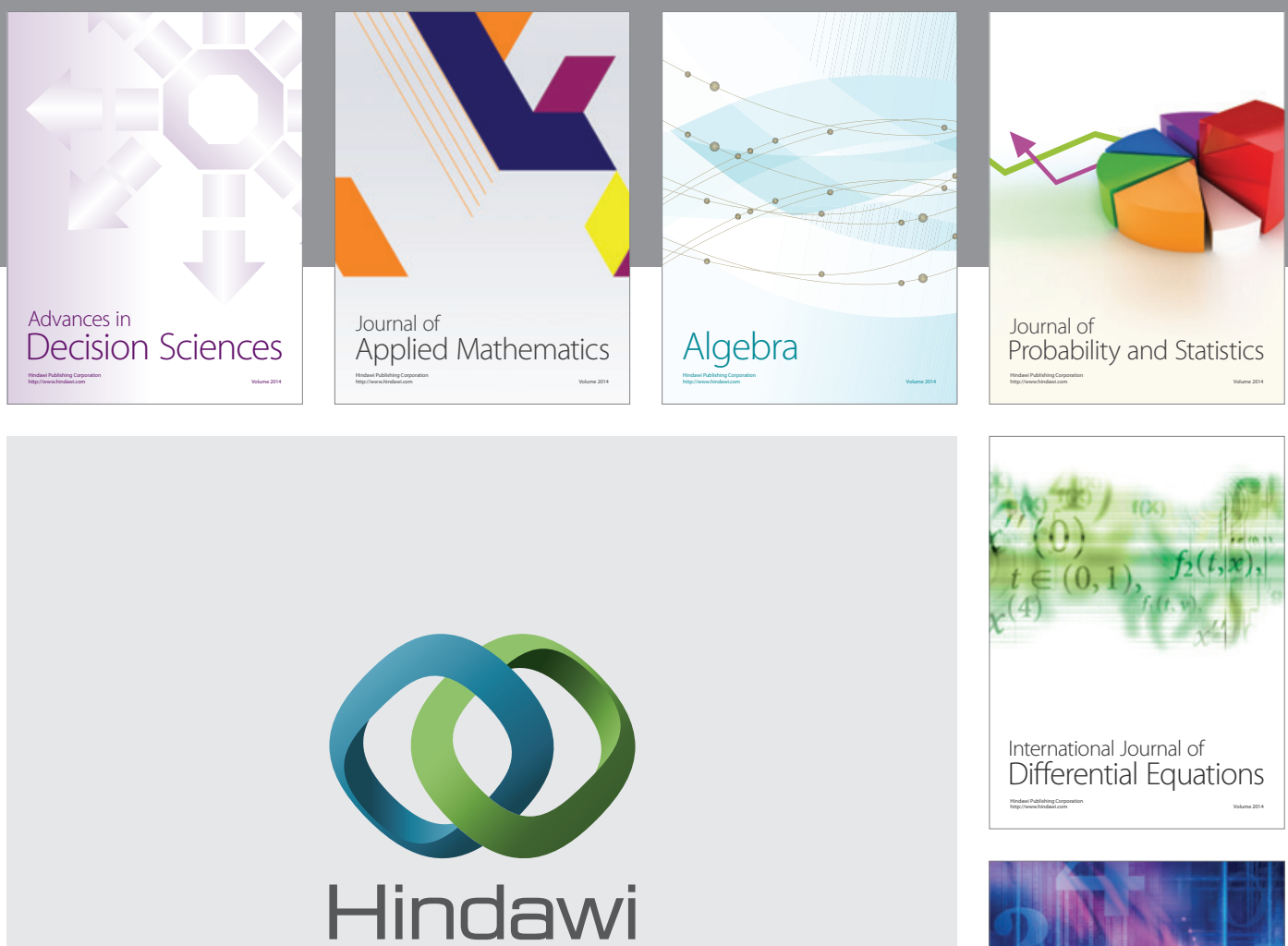

Submit your manuscripts at http://www.hindawi.com
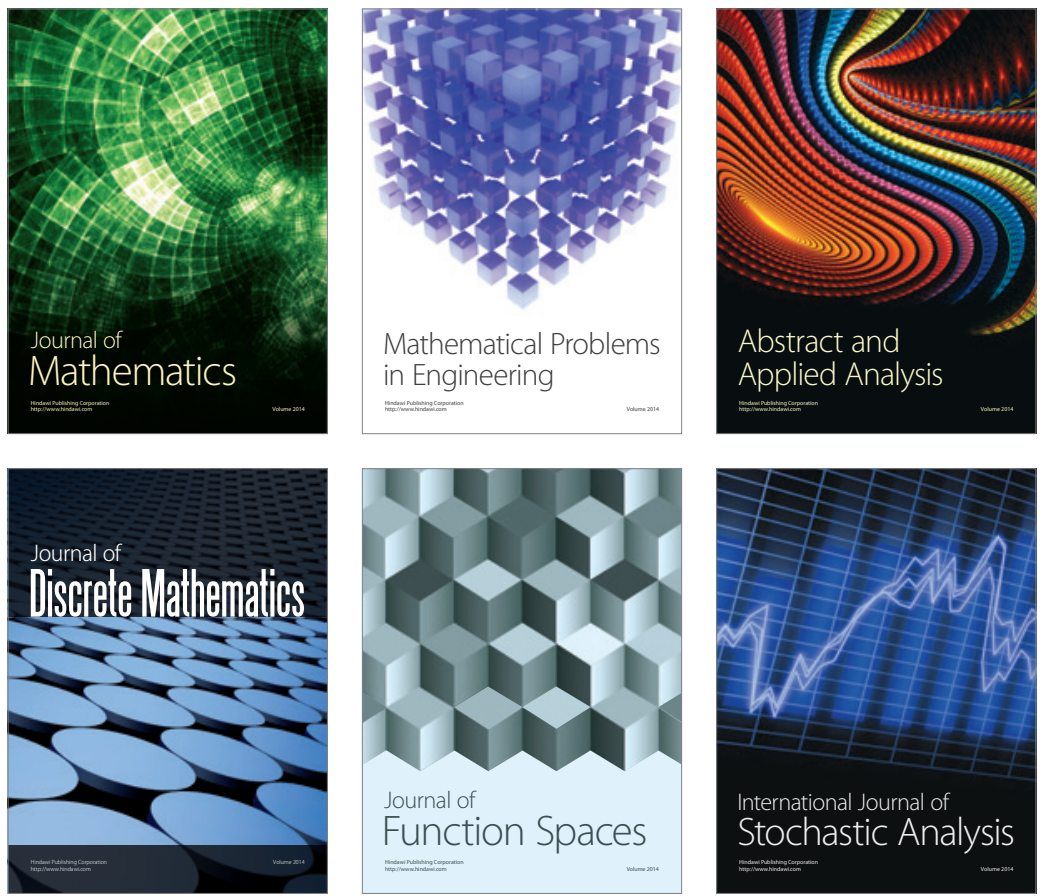

Journal of

Function Spaces

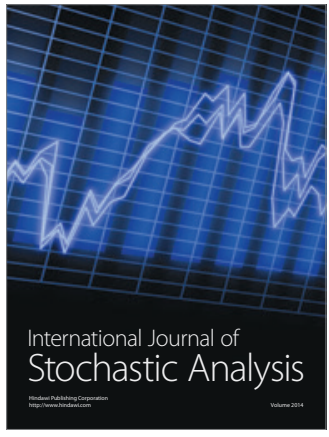

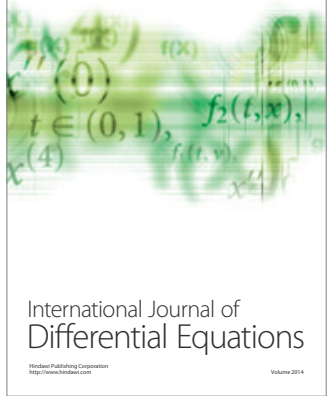
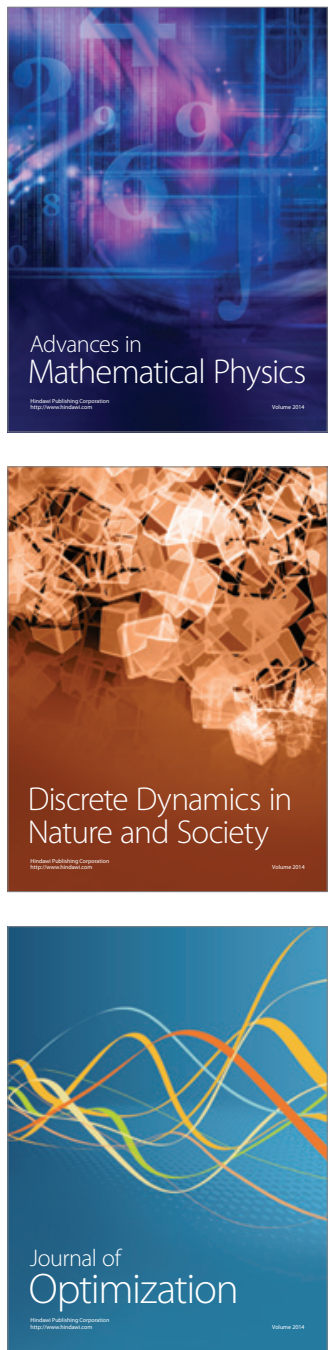